\title{
Directed Paths in a Wedge
}

\author{
E J Janse van Rensburg $₫$, T Prellberg ${ }^{\ddagger}$, A Rechnitzer ${ }^{\star}$, \\ † Department of Mathematics and Statistics, York University, 4700 Keele Street, \\ Toronto, Ontario, M3J 1P3, Canada (rensburg@yorku.ca) \\ ¥ School of Mathematical Sciences, Queen Mary, University of London, Mile End \\ Road, London E1 4NS, UK (t.prellberg@qmul.ac.uk) \\ $\star$ Department of Mathematics, The University of British Columbia, Vancouver, \\ B.C. V6T 1 Z2 (andrewr@math.ubc.ca)
}

\begin{abstract}
Directed paths have been used extensively in the scientific literature as a model of a linear polymer. Such paths models in particular the conformational entropy of a linear polymer and the effects it has on the free energy. These directed models are simplified versions of the self-avoiding walk, but they do nevertheless give insight into the phase behaviour of a polymer, and also serve as a tool to study the effects of conformational degrees of freedom in the behaviour of a linear polymer. In this paper we examine a directed path model of a linear polymer in a confining geometry (a wedge). The main focus of our attention is $c_{n}$, the number of directed lattice paths of length $n$ steps which takes steps in the North-East and South-East directions and which is confined to the wedge $Y= \pm X / p$, where $p$ is an integer. In this paper we examine the case $p=2$ in detail, and we determine the generating function using the iterated kernel method. We also examine the asymtotics of $c_{n}$. In particular, we show that

$$
c_{n}=[0.67874 \ldots] \times 2^{n-1}\left(1+(-1)^{n}\right)+O\left(\left(4 / 3^{3 / 4}\right)^{n+o(n)}\right)+o\left(\left(4 / 3^{3 / 4}\right)^{n}\right)
$$

where we can determine the constant $0.67874 \ldots$ to arbitrary accuracy with little effort.

PACS numbers: 05.50.+q, 02.10.Ab, 05.40.Fb, 82.35.-x
\end{abstract}

Submitted to: $J P A$

$\S$ To whom correspondence should be addressed (rensburg@yorku.ca) 


\section{Introduction}

Lattice paths and lattice walks have long been used as models of conformational entropy in linear polymers 9, 10. Perhaps the most famous of these models is the self-avoiding walk [22, 16]. The most fundamental quantity in this model is $w_{n}$, the number of self-avoiding walks of length $n$ steps from the origin in the hypercubic lattice. Is it known that $w_{n}=\mu^{n+o(n)}$, where $\mu$ is the growth constant of the selfavoiding walk, and that the $\operatorname{limit}_{\lim } \lim _{n \rightarrow \infty} w_{n}^{1 / n}=\mu$ exists [12]. The self-avoiding walk is a non-Markovian model, and it is generally very difficult to extract its properties by rigorous or even by numerical means.

Directed paths, in particular models in two dimensions, are generally recurrent models which can often be solved exactly by a renewal type argument. A particular example is the Dyck path which renews itself each time its visits the line $Y=X$ in the square lattice. This property introduces a translational invariance in the model, which can be used directly to solve for the generating function as a root of a quadratic polynomial, see for example reference [8]. This general observation holds for other models, including a model of directed paths above the line $Y=2 X[11$ and partially directed lattice paths including bargraph paths [3, 4, 25, 26, 27] and other related models such as Motzkin paths [5].

Confining a lattice path to a wedge in the square lattice introduces complexities which oftens makes the model harder to solve. These models are directed versions of the self-avoiding walk confined to a wedge [13, which in turn is a model of a linear polymer in a confined geometry. A Dyck path is perhaps the simplest directed path model of a path confined in a wedge. In figure 1(a) a more generic directed path in a wedge formed by the $Y$-axis and the line $Y=r X$ is illustrated (where $r \geq 0$ ). This model can be solved exactly for $r \in\{0,1,2,3\}$, but no explicit solutions are known for other values of $r$ although the radius of convergence of the generating function is known explicity for all $r \geq 0$, see for example references [7, 6, 15, 20, 17, 19. Generally these models pose challenging combinatorial questions. If the line $Y=r X$ has rational slope, then the generating function is a root of polynomial (it is algebraic), and a recurrance can be determined by a renewal type argument: The path renews itself each time its visits the line $Y=r X$. More generally $r$ is irrational, and in these models there is no translational invariance of the model along the line $Y=r X$ and a recurrence relation for the number of paths seems out of the question.

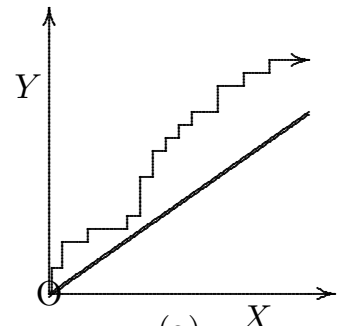

(a)

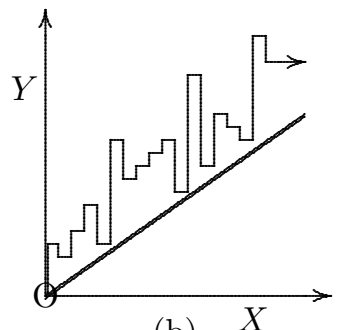

(b)

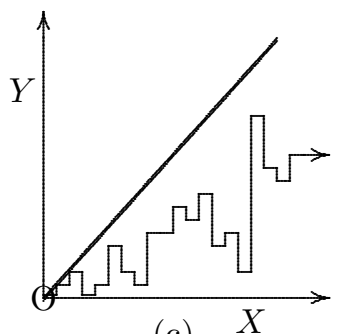

(c)

Figure 1. (a) A directed path in the wedge formed by the $Y$-axis and the line $Y=r X$. (b) A partially directed path in the wedge formed by the $Y$-axis and the line $Y=r X$.

The model in figure 1(a) can be generalised by considering a partially directed path above the line $Y=r X$ as illustrated in figure 1(b). A recurrence relation for the 


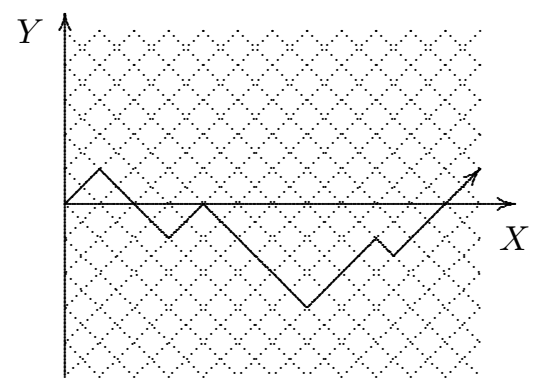

Figure 2. A fully directed path in the square lattice, starting at the origin, and giving steps in the North-East and South-East directions.

generating function of these model has been written down for some values of $r$ [18, but generally these models pose a significant mathematical problem. A third variant of these models is illustrated in figure 1(c). In this case a partially directed path is included in the wedge formed by the $X$-axis and the line $Y=r X$. A model of this type was proposed and solved in the case that $r=1$ in reference [21.

In this paper we generalise models of fully directed paths from the origin to a model of lattice paths in a wedge. Consider the directed path in figure2 2 in the square lattice which takes steps only in the North-East and South-East directions. The most fundamental quantity in this model is $c_{n}$, the number of paths from the origin of length $n$ steps. Obviously, in this model $c_{n}=2^{n}$. This path can be put into a wedge generally as illustrated in figure 3. This is a symmetric wedge (about the $X$-axis), and is an alternative and more challenging model compared to the cases illustrated in figure 1(a).

We are primarily interested in a model of directed paths starting from the vertex $(2,0)$ and confined to the wedge formed by the lines $Y= \pm X / 2$. We determine recurrences for the generating function using the kernel method [24], and in particular a variant of this method developed in reference 21 and which we will call the iterated kernel method.

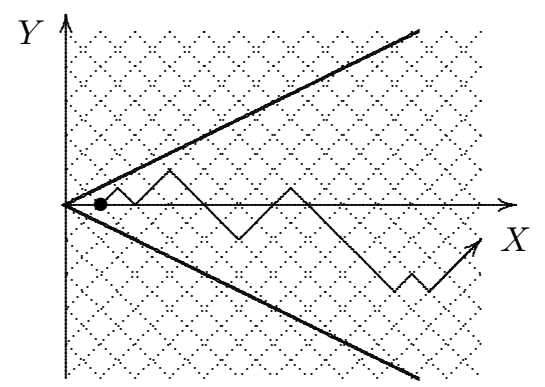

Figure 3. A directed path from the vertex $(2,0)$ in a symmetric wedge formed by the lines $Y= \pm X / 2$.

The most fundamental quantity in our model is $c_{n}$, the number of paths of length $n$ steps and confined to the wedge formed by $Y= \pm X / 2$ and starting from the vertex with coordinates $(2,0)$. We will solve for the generating function of $c_{n}$, and our solution will be an alternating series of compositions of a root of a quartic polynomial. This will 
allow us to determine $c_{n}$ to high accuracy: in particular, we show that asymptotically,

$$
c_{n}=[0.67874 \ldots] \times 2^{n-1}\left(1+(-1)^{n}\right)+O\left(\left(4 / 3^{3 / 4}\right)^{n+o(n)}\right)+o\left(\left(4 / 3^{3 / 4}\right)^{n}\right)
$$

where the constant 0.67874 can be determined to hundreds of significant digits with minimal computational effort.

In section 2 we define our models and determine a recurrence for the generating function. In addition, we note that $\lim _{n \rightarrow \infty} c_{n}^{1 / n}=2$, we solve for the generating function using the kernel method, and examine the properties of the roots of the kernel. These results allows us to compute the constant in equation (11) in section 3 by examining the singularities in the generating function. We conclude the paper with some final comments in section 4 .

\section{Directed Paths in a Wedge}

Let $\mathbb{L}$ be the square lattice of points with integer coordinates in the plane. A directed path in this lattice is a path that takes only North-East (NE) and South-East (SE) steps. If the path consists of $n$ steps (or edges), then there are $2^{n}$ such paths. One such path is illustrated in figure 2, Let $X Y$ be the usual Cartesian coordinate system in figure 2, with origin at the first vertex of the path. Then the edges in the directed path have length $\sqrt{2}$ each.

The directed path in figure 2 is unconstrained by the boundaries of the wedge $Y= \pm X$. This model becomes more interesting if the path is constrained by a narrower wedge $Y= \pm X / p$, where $p \geq 1$ is an integer.

Define the $1 / p$-wedge $V_{p}$ by

$$
V_{p}=\{(x, y) \in \mathbb{L} \mid \text { where }-x / p \leq y \leq x / p\} .
$$

Then $V_{p}$ is the subset of $\mathbb{L}$ in the first and fourth quadrants bounded by the lines $Y= \pm X / p$. In figure 3 a directed path confined to the wedge $V_{p}$ is denoted where $p=2$. This path has its first vertex at the point with coordinates $(2,0)$. Generally, directed paths in the wedge $Y= \pm X / p$ will have their first vertices at $(p, 0)$.

\subsection{Directed paths in the Wedge formed by $Y= \pm X / 2$}

In this section we determine a recurrance for the generating function $G_{2}$ of directed paths giving $N E$ and $S E$ steps from the vertex $(2,0)$ in the wedge $V_{2}$. Proceed by introducing the edge generating variable $t$ and define

$$
G_{2}(a, b)=\sum_{n, u, v \geq 0} c_{n}^{(2)}(u, v) u^{a} v^{b} t^{n}
$$

where $c_{n}^{(2)}(u, v)$ is the number of directed paths in the wedge $V_{2}$ from the vertex $(2,0)$ of length $n$ and with final vertex a vertical distance $u$ from $Y=\lceil X / 2\rceil$ and a vertical distance $v$ from $Y=-\lceil X / 2\rceil$.

Observe that the unit of vertical distance is determined by the fact that the squares in figure 3 have diagonal length 2. For example, a NE-step will increase the $Y$-coordinate of the endpoint by 1 , and the distance between the endpoint and $Y=\lceil X / 2\rceil$ may either decrease by 1 if the initial $X$-coordinate is even, or remain unchanged otherwise.

Consider the examples in figure 4. The vertical distances are measured to the step-functions $Y= \pm\lceil X / 2\rceil$, and in these examples the vertical distances are $u=11$ 
and $v=7$ (figure 4(a)) and $u=13$ and $v=7$ (figure 4(b)). In addition, $u+v$ is always even.

We are fundamentally interested in $c_{n}^{(2)}=\sum_{u, v>0} c_{n}^{(2)}(u, v)$, the number of paths of length $n$ in the wedge $V_{2}$. The generating variables $a$ and $b$ are introduced to account for the vertical distance of the endpoint from the wedge boundaries and to enable us to determine $G_{2}(1,1)$.

The examples of paths in figure 4 illustrates that the enumeration of these paths are fundamentally affected by a parity effect. Paths of even length (figure 4 (a)) are either the single vertex at the point $X=2$ generated by $a b$, or can be generated by adding a $N E$-step or $S E$-step to a path of odd length. Paths of odd length can only be generated by extending a path of even length by the addition of a $N E$-step or a $S E$-step.

Let $g_{0}(a, b)$ generate paths of even length, and suppose that $g_{1}(a, b)$ generates paths of odd length. Then

$$
G_{2}(a, b)=g_{0}(a, b)+g_{1}(a, b) .
$$

Thus, by determining $g_{0}(a, b)$ and $g_{1}(a, b), G_{2}(a, b)$ can be determined.

Recurrences for $g_{0}(a, b)$ and $g_{1}(a, b)$ can be obtained by arguing as in figures 5 and 6. The basic idea is to create paths generated by $g_{1}(a, b)$ by appending an edge to paths of even length generated by $g_{0}(a, b)$, and to create paths generated by $g_{0}(a, b)$ by appending an edge to paths generated by $g_{1}(a, b)$. The resulting recurrances are

$$
\begin{aligned}
& g_{0}(a, b)=a b+t(a / b+b / a) g_{1}(a, b)-t(a / b) g_{1}(a, 0)-t(b / a) g_{1}(0, b), \\
& g_{1}(a, b)=t\left(a^{2}+b^{2}\right) g_{0}(a, b)-t a^{2} g_{0}(a, 0)-t b^{2} g_{0}(0, b) .
\end{aligned}
$$

These recurrences can be iterated to enumerate the directed paths. The numbers for even length paths are given in table 2.1.

The number of digits in the $c_{n}$ in table 2.1 increases linearly with $n$, which implies exponential growth of $c_{n}$. It is in fact possible to prove explicitly that $c_{n}$ increases proportional to $2^{n}$, using the techniques in reference [13. In addition, since $c_{n} c_{m} \leq c_{n+m}$ (concatenate a path of length $m$ with a path of length $n$ by translating the first until its first vertex coincide with the last vertex of the second to see this), $c_{n}$ is a supermultiplicative function of $n$, and thus the limit

$$
\lim _{n \rightarrow \infty} c_{n}^{1 / n}=2
$$

exists 14. Thus, there is a function of $n, C_{0}=e^{o(n)}$, such that

$$
c_{n}=C_{0} 2^{n}+o\left(2^{n}\right) .
$$

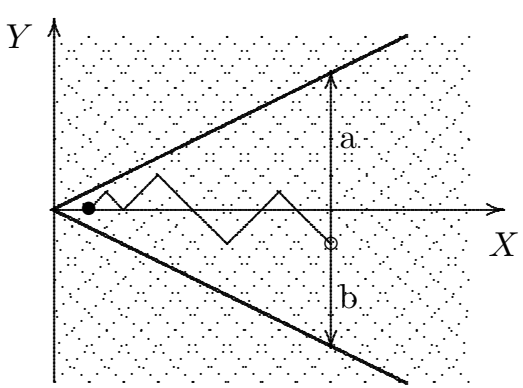

(a)

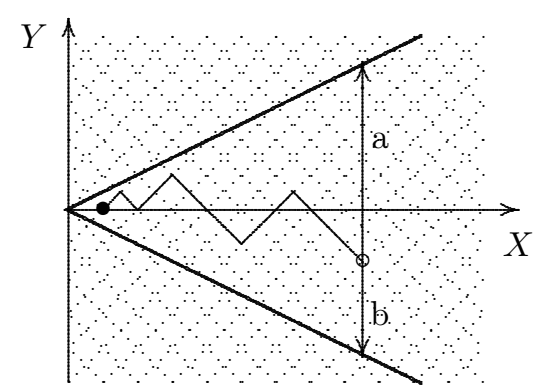

(b)

Figure 4. a symmetric wedge formed by the lines $Y= \pm X / 2$. 

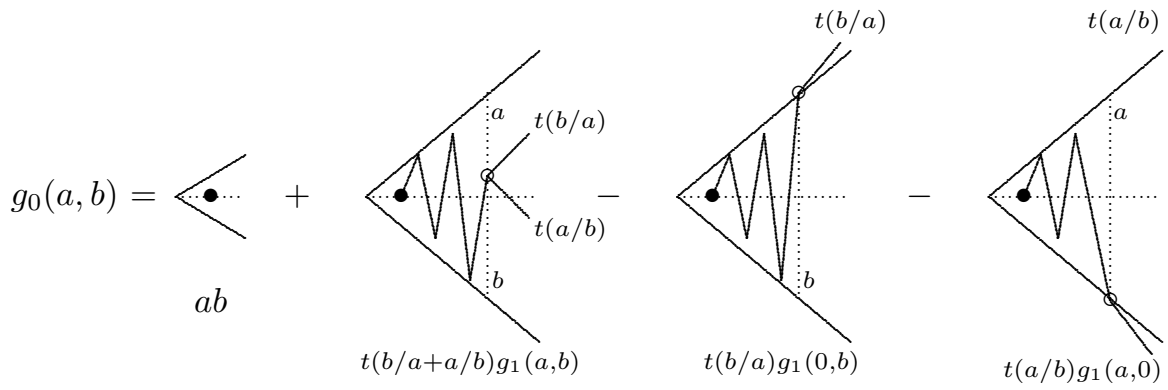

Figure 5. Determining $g_{0}(a, b)$. Each path generated by $g_{0}(a, b)$ is either the single vertex at $(2,0)$ generated by $a b$, or it is generated by appending a $N E$ edge onto a path of odd length (this gives the term $(t a b)(b / a) g_{1}(a, b)$ ), or it is generated by appending a $S E$-edge onto a path of odd length (giving the term $\left.(t a b)(a / b) g_{1}(a, b)\right)$. Lastly, paths which step outside the wedge must be subtracted: $(t a b)(b / a) g_{1}(0, b)$ if the path steps over the line $Y=X / 2$ and $(t a b)(a / b) g_{1}(a, 0)$ if the path steps over the line $Y=-X / 2$.

Examination of the data in table 2.1 shows that $c_{n} / 2^{n}$ approaches a constant. Assuming that $C_{0}$ is a constant, one may estimate it numerically. Dividing $c_{n}$ by $2^{n}$ and increasing $n$ up to $n=82$ gives $C_{0}$ to five digits, namely

$$
C_{0}=0.67874 \ldots
$$

We proceed next by computing $C_{0}$ by solving the recurrances in equations (5) above, and by examining the singularities in $g_{0}(a, b)$.

\subsection{Solving equations (5)}

In this section we use the iterated kernel method (see reference [21]) to find an expression for the generating function $g_{0}(a, b)$. Simplify the recurrences by introducing

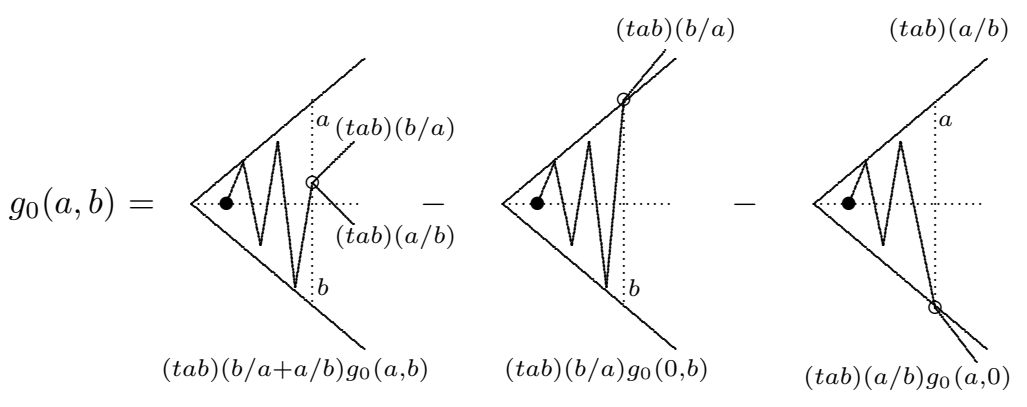

Figure 6. Determining $g_{1}(a, b)$. Each path generated by $g_{1}(a, b)$ is obtained by appending a $N E$-edge or $S E$-edge onto a path of even length. In this case, the vertical distance to the wedge does not increase by a full step, and thus no new factors of $a$ or $b$ are included, apart from accounting for stepping closer to the top boundary if a $N E$-edge is added, or stepping closer to the bottom boundary if a $S E$-edge is added. Thus it generates the term $t(a / b+b / a) g_{0}(a, b)$ as indicated above. Lastly, paths which step outside the wedge must be subtracted: $t(b / a) g_{0}(0, b)$ if the path steps over the line $Y=X / 2$ and $t(a / b) g_{0}(a, 0)$ if the path steps over the line $Y=-X / 2$. 


\begin{tabular}{||c|c||c|c||}
\hline$n$ & $c_{n}$ & $n$ & $c_{n}$ \\
\hline 0 & 1 & 42 & 2985401474160 \\
2 & 4 & 44 & 11941093593120 \\
4 & 12 & 46 & 47764374372480 \\
6 & 48 & 48 & 191053247884320 \\
8 & 180 & 50 & 764212991537280 \\
10 & 720 & 52 & 3056816328436200 \\
12 & 2820 & 54 & 12227265313744800 \\
14 & 11280 & 56 & 48908759609676540 \\
16 & 44760 & 58 & 195635038438706160 \\
18 & 179040 & 60 & 782537580134560920 \\
20 & 713760 & 62 & 3130150320538243680 \\
22 & 2855040 & 64 & 12520579171583415840 \\
24 & 11403060 & 66 & 50082316686333663360 \\
26 & 45612240 & 68 & 200329075631136029040 \\
28 & 182321460 & 70 & 801316302524544116160 \\
30 & 729285840 & 72 & 3205263549296411867340 \\
32 & 2916160800 & 74 & 12821054197185647469360 \\
34 & 11664643200 & 76 & 51284202287042290859820 \\
36 & 46650808680 & 78 & 205136809148169163439280 \\
38 & 186603234720 & 80 & 820547109423871153955280 \\
40 & 746350368540 & 82 & 3282188437695484615821120 \\
\hline
\end{tabular}

Table 1. The number of directed paths in $V_{2}$.

$K=t(a / b+b / a)$ and $L=t\left(a^{2}+b^{2}\right)$. Observe also that $g_{0}(a, 0)=g_{0}(0, a)$, and that $g_{1}(a, 0)=g_{1}(0, a)$.

Since paths of odd length generated by $g_{1}(a, b)$ cannot intersect the boundaries of the wedge, they always will be weighted by factors of $a$ and $b$. Hence, one expects $g_{1}(a, 0)=g_{1}(0, b)=0$ identically. Ignoring this last observation for the moment gives the recurrances in slightly simplified form:

$$
\begin{aligned}
& g_{0}(a, b)=a b+K g_{1}(a, b)-t(a / b) g_{1}(a, 0)-t(b / a) g_{1}(b, 0), \\
& g_{1}(a, b)=L g_{0}(a, b)-t a^{2} g_{0}(a, 0)-t b^{2} g_{0}(b, 0) .
\end{aligned}
$$

Substitute these equations into one another, and write them in kernel form. This gives

$$
\begin{aligned}
& (1-K L) g_{0}(a, b) \\
& \quad=a b-t a^{2} K g_{0}(a, 0)-t b^{2} K g_{0}(b, 0)-t(a / b) g_{1}(a, 0)-t(b / a) g_{1}(b, 0), \\
& (1-K L) g_{1}(a, b) \\
& \quad=L a b-t a^{2} g_{0}(a, 0)-t b^{2} g_{0}(b, 0)-t(a / b) L g_{1}(a, 0)-t(b / a) L g_{1}(b, 0) .
\end{aligned}
$$

We identify the kernel $(1-K L)$ in these equations. Generally, we say that recurrence equations are in kernelized form if the generating function and its coefficients have been collected on the left hand side, while all other terms and boundary terms are on the right hand side. The kernel $(1-K L)$ may be simplified, and then the quartic polynomial

$$
t^{2}\left(a^{2}+b^{2}\right)^{2}-a b
$$

in $a$ and $b$ appears as a factor. 
To proceed, consider this to be a quartic in $b$ with $a$ and $t$ two parameters. To solve the original recurrances, the roots of this quartic must be determined. Closer examination shows that the four roots have the following properties: The first is $\beta_{0}(a)$, which is a power-series in $t$ :

$$
\beta_{0}(a)=a^{3} t^{2}+2 a^{7} t^{6}+9 a^{11} t^{10}+52 a^{15} t^{14}+\ldots
$$

The second real root is $\beta_{1}(a)$, which is singular at $t=0$ :

$$
\beta_{1}(a)=\frac{a^{1 / 3}}{t^{2 / 3}}-\frac{2 a^{5 / 3} t^{2 / 3}}{3}-\frac{28 a^{13 / 3} t^{10 / 3}}{81}+\ldots
$$

while the two remaining roots are a complex conjugate pair:

$$
\beta_{ \pm}(a)=-\frac{a^{1 / 3}(1 \mp i \sqrt{3})}{2 t^{2 / 3}}+\frac{a^{5 / 3} t^{2 / 3}(1 \pm i \sqrt{3})}{3}-\frac{a^{3} t^{2}}{3}+\ldots
$$

Substituting $b=\beta_{0}(a) \equiv \beta_{0}$ in the kernelized equations in (11) and (12) produces two equations for $g_{0}(a, 0), g_{0}(b, 0), g_{1}(a, 0)$ and $g_{1}(b, 0)$ :

$$
\begin{aligned}
& K\left(t a^{2} g_{0}(a, 0)+t \beta_{0}^{2} g_{0}\left(\beta_{0}, 0\right)\right)+\left(t\left(a / \beta_{0}\right) g_{1}(a, 0)+t\left(\beta_{0} / a\right) g_{1}\left(\beta_{0}, 0\right)\right)=a \beta_{0}, \\
& \left(t a^{2} g_{0}(a, 0)+t \beta_{0}^{2} g_{0}(b, 0)\right)+L\left(t\left(a / \beta_{0}\right) g_{1}(a, 0)+t\left(\beta_{0} / a\right) g_{1}\left(\beta_{0}, 0\right)\right)=L a \beta_{0} .
\end{aligned}
$$

The solution of this linear system is

$$
\begin{aligned}
t a^{2} g_{0}(a, 0)+t \beta_{0}^{2} g_{0}\left(\beta_{0}, 0\right) & =L a \beta_{0}, \\
t\left(a / \beta_{0}\right) g_{1}(a, 0)+t\left(\beta_{0} / a\right) g_{1}\left(\beta_{0}, 0\right) & =0 .
\end{aligned}
$$

From the second of these it follows that

$$
g_{1}\left(\beta_{0}(a), 0\right)=-\frac{a^{2}}{\beta_{0}^{2}} g_{1}(a, 0)
$$

and since this generating function cannot be negative, the conclusion is that

$$
g_{1}(a, 0)=0
$$

identically, as claimed above. The first solution above gives

$$
g_{0}(a, 0)=\frac{L\left(a, \beta_{0}\right) \beta_{0}}{t a}-\frac{\beta_{0}^{2}}{a^{2}} g_{0}\left(\beta_{0}, 0\right) .
$$

Proceed by defining $\beta^{(n)}(a)=\left(\beta_{0} \circ \beta_{0} \circ \ldots \circ \beta_{0}\right)(a)$ to be the composition of $\beta_{0}$ $n$-times with itself. Define $\beta^{(0)}(a)=a$, then the last equation may be written as

$$
g_{0}\left(\beta^{(n-1)}, 0\right)=\frac{L\left(\beta_{0}^{(n-1)}, \beta_{0}^{(n)}\right) \beta_{0}^{(n)}}{t \beta_{0}^{(n-1)}}-\left(\frac{\beta_{0}^{(n)}}{\beta^{(n-1)}}\right)^{2} g_{0}\left(\beta^{(n)}, 0\right) .
$$

This may be iterated to obtain a solution for $g_{0}(a, 0)=g_{0}\left(\beta_{0}^{(0)}, 0\right)$ :

$$
g_{0}(a, 0)=\frac{1}{t a^{2}} \sum_{n=0}^{\infty}(-1)^{n} L\left(\beta_{0}^{(n)}\right) \beta_{0}^{(n)} \beta_{0}^{(n+1)}
$$

Thus, one may solve for the generating function $g_{0}(a, b)$ from the kernelized equations in (11) and (12):

$$
g_{0}(a, b)=\frac{a b-t K\left(a^{2} g_{0}(a, 0)+b^{2} g_{0}(b, 0)\right)}{1-K L}
$$

and the radius of convergence is given by the dominant root of the quartic in equation (13), provided that $g_{0}(a, 0)$ does not have compensating singularities at the same points. 
2.3. More on the roots of $t^{2}\left(a^{2}+b^{2}\right)^{2}-a b$.

One may check that

$$
\beta_{0}(a)=\left[2 t^{2} a^{3}\right] \sum_{n=0}^{\infty}\left(\begin{array}{c}
4 n+1 \\
n
\end{array}\right) \frac{(a t)^{4 n}}{3 n+2}
$$

as given in equation (14). The radius of convergence of this series is $|a t|^{4}=3^{3} / 4^{4}$.

Closer examination also shows that $\beta_{0}(a)$ counts directed paths with first step in the SE-direction, above the line $Y=-X / 2$ and with last vertex in the line $Y=-X / 2$. The root $\beta_{1}$ is the inverse function of $\beta_{0}$ : Direct calculation shows that $\beta_{0} \circ \beta_{1}(a)=\beta_{1} \circ \beta_{0}(a)=a$.

The roots $\beta_{+}$and $\beta_{-}$are not independent, but one may check that

$$
\beta_{+}\left(\frac{i x^{3}}{8}\right)=-\beta_{-}\left(\frac{-i x^{3}}{8}\right)=-\frac{i x}{2 t^{2 / 3}}+\frac{i t^{2 / 3} x^{5}}{48}+\frac{i t^{2} x^{9}}{1536}+\frac{7 i t^{10 / 3} x^{13}}{165888}+\ldots
$$

Solving for the roots of the quartic is equivalent to solving the non-linear system

$$
\begin{aligned}
& r^{2}=\left(a^{2}+b^{2}\right), \\
& t^{2} r^{4}=a b
\end{aligned}
$$

for $a$ and $b$. There are four solutions, two given by the pairs $\left(a_{1}(r), b_{1}(r)\right)$ and $\left(-a_{1}(r),-b_{1}(r)\right)$, where

$$
\begin{aligned}
& a_{1}(r)=\frac{\sqrt{2} r^{3} t^{2}}{\sqrt{1+\sqrt{1-4 t^{4} r^{4}}}}=\frac{r \sqrt{1-\sqrt{1-4 t^{4} r^{4}}}}{\sqrt{2}} \\
& b_{1}(r)=\frac{r \sqrt{1+\sqrt{1-4 t^{4} r^{4}}}}{\sqrt{2}}=\frac{\sqrt{2} r^{3} t^{2}}{\sqrt{1-\sqrt{1-4 t^{4} r^{4}}}},
\end{aligned}
$$

and two more given by the pairs $\left(b_{1}(r), a_{1}(r)\right)$ and $\left(-b_{1}(r),-a_{1}(r)\right.$ ) (where we interchanged $a_{1}$ and $b_{1}$ ). An expression for $r^{2}$ is given below in equation (35). One may check as well that

$$
\begin{aligned}
& a_{1}(r)=t^{2} r^{3}\left(1+t^{4} r^{4} \sum_{n=0}^{\infty}\left(\begin{array}{c}
4 n+3 \\
2 n
\end{array}\right) \frac{(t r)^{4 n}}{(2 n+1) 4^{n+1 / 2}}\right) \\
& b_{1}(r)=r\left(1-t^{4} r^{4} \sum_{n=0}^{\infty}\left(\begin{array}{c}
4 n+1 \\
2 n
\end{array}\right) \frac{(t r)^{4 n}}{(2 n+2) 4^{n}}\right)
\end{aligned}
$$

The roots of the quartic may be found by inverting $a_{1}$ to obtain $r_{a}(a)$ so that $r_{a} \circ a_{1}=a_{1} \circ r_{a}$ is the identity map. Then $\beta_{0}(a)=b_{1} \circ r_{a}$. Inverting $b_{1}$ to obtain $r_{b}(b)$ gives a second root by the composition $\beta_{1}=a_{1} \circ r_{b}$. In particular, this means for example that $\beta_{0} \circ \beta_{1}=b_{1} \circ\left(r_{a} \circ a_{1}\right) \circ r_{b}=b_{1} \circ r_{b}=$ identity since $a_{1}^{-1}=r_{a}$ and $b_{1}^{-1}=r_{b}$. This proves the observation above that $\beta_{0} \circ \beta_{1}=\beta_{1} \circ \beta_{0}$ is the identity map. In other words, the composition of two roots of the quartic is the identity.

The other two roots of the quartic is given by the compositions $b_{1} \circ r_{b}$ and $a_{1} \circ r_{a}$. Unfortunately, while explicit expressions for $r_{a}$ and $r_{b}$ can be obtained, they are rather lengthy; both $r_{a}^{2}$ and $r_{b}^{2}$ are roots of the quartic $t^{4} x^{4}-c^{2} x+c^{4}$ where $c=a$ for $r_{a}$ and $c=b$ for $r_{b}$. This may be examined by iteration to determine the first few terms in $r_{a}^{2}$. Comparison of the results to the online encyclopedia of integers [28] shows that

$$
r_{a}^{2}(a)=a^{2} \sum_{n=0}^{\infty}\left(\begin{array}{c}
4 n \\
n
\end{array}\right) \frac{(a t)^{4 n}}{3 n+1} .
$$


The second root of $t^{4} x^{4}-c^{2} x+c^{4}$ proposes the "unphysical" series starting with $\left(a / t^{2}\right)^{2 / 3}+O\left(a^{2}\right)$ for $r_{a}^{2}(a)$. The series expression for $r_{a}^{2}(a)$ may finally be substituted in $b_{1}(r)$ to obtain an expression for the root $\beta_{0}(a)$ of the quartic:

$$
\beta_{0}(a)=\frac{r_{a}(a) \sqrt{1+\sqrt{1-4 t^{4} r_{a}^{4}(a)}}}{\sqrt{2}} .
$$

Remarkably, this evaluates to equation (27) and compositions of this with itself will eventually lead to the expression for $g_{0}(a, 0)$ in equation (25).

In addition, having determined $\beta_{0}(a)$, one may consider the composition of $a_{1}(r)$ and $r_{a}(a)$, which is

$$
\frac{r_{a}(a) \sqrt{1-\sqrt{1-4 t^{4} r_{a}^{4}(a)}}}{\sqrt{2}}=a,
$$

and which must be the identity map. In other words, by appealing to equation (30) it follows that

$$
a \cdot \beta_{0}(a)=t^{2} r_{a}(a)^{4},
$$

and from equation (27) one concludes that the identity

$$
\left[\sum_{n=0}^{\infty}\left(\begin{array}{c}
4 n \\
n
\end{array}\right) \frac{(a t)^{4 n}}{3 n+1}\right]^{2}=2 \sum_{n=0}^{\infty}\left(\begin{array}{c}
4 n+1 \\
n
\end{array}\right) \frac{(a t)^{4 n}}{3 n+2}
$$

should be closely related to the combinatorial properties of directed paths in the wedge $V_{2}$.

\section{Determining $C_{0}$.}

In this section we examine the generating function $g_{0}(a, b)$ in equation (26) with $a=b=1$. Singularities in this generating function arise from several possible sources. In the first instance there are simple poles at the zeros of the kernel $(1-K L)$ in the denominator. These are located at $t= \pm 1 / 2$. In order to determine the constant $C_{0}$, we examine the residue of $g_{0}(1,1) / t^{n+1}$ at $t= \pm 1 / 2$.

Put $a=1$ and $t=1 / 2$ in $\beta_{0}(a)$. Compositions of $\beta_{0}(a)$ with itself at this point gives the following values

$$
\begin{aligned}
& \beta_{0}^{(0)}(1)=1 \\
& \beta_{0}^{(1)}(1)=2.9559774252208477098 \ldots \times 10^{-1} \\
& \beta_{0}^{(2)}(1)=6.4633625443847777820 \ldots \times 10^{-3} \\
& \beta_{0}^{(3)}(1)=6.7501832073150278963 \ldots \times 10^{-8} \\
& \beta_{0}^{(4)}(1)=7.6892979457392165146 \ldots \times 10^{-23} \\
& \beta_{0}^{(5)}(1)=1.1365801752937162161 \ldots \times 10^{-67} \\
& \beta_{0}^{(6)}(1)=3.6706268625677440729 \ldots \times 10^{-202}
\end{aligned}
$$

where explicitly

$$
\beta_{0}(1)=\frac{5-\sqrt{33}}{12}(19+3 \sqrt{33})^{2 / 3}+\frac{\sqrt{33}-1}{12}(19+3 \sqrt{33})^{1 / 3}-\frac{1}{3},
$$


and the other terms are more complicated expressions involving nested radicals which do not simplify to manageable expressions.

Generally, we observe that $\beta_{0}^{(n)}(1)=\alpha_{n}$ at $t=1 / 2$, and one may check that $\alpha_{n+1} \approx \alpha_{n}^{3} / 4$. For example, $\alpha_{3}^{3} / 4=7.689297941 \ldots \times 10^{-23} \approx \alpha_{4}$. This observation follows from the expansion $\beta_{0}(a)=a^{3} t^{2}+O\left(a^{7} t^{6}\right)$ so that compositions of $\beta_{0}$ at $t=1 / 2$ and $a=1$ quickly converges to zero. In other words, the recurrence $x_{n+1}=\left.\beta_{0}\left(x_{n}\right)\right|_{t=1 / 2}$ is a fixed point iteration of order three. This fast convergence allows the accurate numerical estimation of $\beta_{0}^{(n)}(1)$ at $t=1 / 2$.

At $a=b=1$ the expression of $g_{0}(1,1)$ in terms of $g_{0}(1,0)$ and $g_{0}(0,1)$ is given by,

$$
g_{0}(1,1)=\frac{1-2 t^{2}\left(g_{0}(1,0)+g_{0}(0,1)\right)}{1-4 t^{2}} .
$$

where

$$
g_{0}(1,0)=g_{0}(0,1)=\sum_{n=0}^{\infty}(-1)^{n} L\left(\beta_{0}^{(n)}\right) \beta_{0}^{(n)} \beta_{0}^{(n+1)},
$$

and where $L(a)=a^{2}+\beta_{0}^{2}$. Numerical evaluation of the residue at $t=1 / 2$ using the calculated values of $\beta^{(n)}(1)$ above, gives the leading order behaviour of the number of paths of length $n$ :

$$
c_{n}=2^{n-1} \times 0.6787405307981094574172327 \ldots+\text { parity term }+\ldots
$$

where the seven values of $\beta_{0}^{(n)}(1)$ listed above produces $C_{0}$ accurately up to at least $O\left(10^{-401}\right)$ or 400 digits if each is calculated to at least this accuracy by using equations (38) or (27) or by explicitly using the closed form expression for $\beta_{0}(a)$ and computing it to high accuracy using a symbolic computations package such as Maple.

To determine the parity effects, we determine the residue at the pole located at $t=-1 / 2$ by repeating the analysis above. Put $a=1$ and $t=-1 / 2$ in $\beta_{0}(a)$. Compositions of $\beta_{0}(a)$ with itself at this point gives the identical values obtained above for $t=1 / 2$. Thus, we conclude that

$$
c_{n}=2^{n-1}\left(1+(-1)^{n}\right) \times(0.6787405307981094574172327 \ldots)+\ldots
$$

This is not an unexpected result since $g_{0}(1,1)$ should only enumerate even length paths; we note that $c_{2 n+1}=0$ in the generating function $g_{0}(1,1)$. This result verifies the numerical estimate for $C_{0}$ from the data in table 2.1 in equation (8).

Contributions to $c_{n}$ also arise from singularities in the numerator in equation (26). In particular, there are branch points and possibly other singularities in $g_{0}(1,0)$ and $g_{0}(0,1)$, and these are due to branch points in $\beta_{0}$.

The radius of convergence of $\beta_{0}$ can be determined when $a=1$ by examining equations (35) and (38) with $a=1$. In particular, $r_{1}(1)$ in equation (35) is convergent for all $|t| \leq 3^{3 / 4} / 4$; in fact, evaluation shows directly that $r_{1}(1)$ is convergent for $|t|=3^{3 / 4} / 4$. Since $3^{3 / 4} / 4>1 / 2$, this proves that the simple poles at $t= \pm 1 / 2$ are within the radius of convergence of $\beta_{0}(1)$.

Further examination of $\beta_{0}(a)$ by Maple shows a complicated expression of nested radicals which expicitly contains factors of the form $\sqrt{81-768 a^{4} t^{4}}$. This shows that there are branch points at $a t=3^{3 / 4} \omega / 4$ where $\omega$ is a fourth root of unity. There may be more branchpoints on the circle $a t=3^{3 / 4} / 4$, but we did not verify this, and this will not play a crucial role in what follows. 
Next, we consider the compositions of $\beta_{0}$ in the alternating sum definition of $g_{0}(1,0)$. Since $r_{1}(t)$ is a positive term power series it follows by the triangle inequality in equation (35) that for (complex) $t$ such that $|t| \leq 3^{3 / 4} / 4$,

$$
\left|r_{1}^{2}(1)\right| \leq \sum_{n=0}^{\infty}\left(\begin{array}{c}
4 n \\
n
\end{array}\right) \frac{\left(3^{3 / 4} / 4\right)^{4 n}}{3 n+1}=\frac{4}{3} .
$$

Thus, by equation 38

$$
\left|\beta_{0}(1)\right| \leq\left[3^{3 / 4} / 4\right]^{2}(16 / 9)=\frac{1}{\sqrt{3}}, \quad \text { if }|t| \leq 3^{3 / 4} / 4 .
$$

Since $\beta_{0}(a)$ is a power series with positive coefficients in both $a$ and $t,\left|\beta_{0}(a)\right| \leq\left|\beta_{0}(1)\right|$ for any $|t| \leq 3^{3 / 4} / 4$ and $|a| \leq 1$, and by the triangle inequality, $\beta_{0}(a)$ is a maximum when $t=3^{3 / 4} / 4$ for a fixed value of $a$. Thus, for fixed values of $|a| \leq 1, \beta_{0}(a)$ is a maximum on the closed disk $|t| \leq 3^{3 / 4} / 4$ when $t=3^{3 / 4} / 4$.

The above shows that for $t=3^{3 / 4} / 4,\left|\beta_{0}(a)\right| \leq 1 / \sqrt{3}$ for $|a| \leq 1$. Moreover, it follows from equation (27) that $\left|\beta_{0}(a)\right| \leq|a| / \sqrt{3}$ for $|a| \leq 1$ when $t=3^{3 / 4} / 4$. In other words,

$$
\left|\beta_{0}\left(\beta_{0}(1)\right)\right| \leq\left|3^{3 / 4} / 4 \sqrt{3}\left(\sqrt{3} \cdot 3^{3 / 4} / 4\right)\right|=\frac{1}{\sqrt{3}^{2}} .
$$

It follows inductively that

$$
\left|\beta_{0}^{(n)}(1)\right| \leq \frac{1}{\sqrt{3}^{n}}
$$

Since the branch points in $\beta_{0}^{(n)}(1)$ will occur at values of $t$ that $\left|\beta_{0}^{(n-1)}(1)\right|=3^{3 / 4} / 4$, and $\left|\beta_{0}^{(n-1)}(1)\right| \leq 1 / \sqrt{3}^{n-1}<3^{3 / 4} / 4$ at $a=1$ and for $n>2$, this implies that the branch points in $\beta^{(n)}(1)$ for $n>1$ lies outside the circle with radius $|t|=3^{3 / 4} / 4$, and contributions of these branch points to $c_{n}$ are dominated by the contributions to $c_{n}$ which is a result of the branch points in $\beta_{0}(1)$ itself.

In particular, since the radius of convergence of $\beta_{0}(a)$ is determined by $|a t|=$ $3^{3 / 4} / 4$ and $\left|\beta_{0}^{(n-1)}(1)\right| \leq 1 / \sqrt{3}^{n-1}$, it follows that the radius of convergence of $\beta_{0}^{(n)}(1)$ in the $t$-plane is on or outside the circle with radius $|t|=3^{3 / 4} \sqrt{3}^{n-1} / 4$, for $n>2$. Thus, the generating function $g_{0}(a, 0)$ has infinitely many singularities in the $t$-plane for $a=1$, and by remark 1 following proposition 9 in reference [2], $g_{0}(1,0)$ cannot be holonomic (or D-finite). Thus, $g_{0}(1,1)$, the generating function of even length directed paths in the wedge formed by $Y= \pm X / 2$, is not holonomic.

The bound in equation (44) also proves that $g_{0}(1,0)$ is an absolutely convergent series on the open disk with radius $|t|=3 \sqrt{3}$ which includes the simple poles of $g_{0}(1,1)$ at $t= \pm 1$ and the branch points on the circle $|t|=3^{3 / 4} / 4$ in its interior. In other words, the asymptotic behaviour of $c_{n}$ is given by

$$
\begin{aligned}
c_{n}= & 2^{n-1}\left(1+(-1)^{n}\right) \times(0.6787405307981094574172327 \ldots) \\
& + \text { corrections. }
\end{aligned}
$$

The corrections are due to the branch points in $\beta_{0}(1)$ and they grow at the exponential rate $\left(4 / 3^{3 / 4}\right)^{n+o(n)}$. Since $4 / 3^{3 / 4} \approx 1.75<2$, the effects of the correction terms will dissappear fast with increasing $n$, and $c_{n} / 2^{n}$ will approach $0.6787405307981094574172327 \ldots$ at an exponential rate with increasing $n$. 


\section{Narrower Wedges}

It is possible to consider this problem in the narrower wedge $V_{p}$ where $a$ and $b$ measure vertical distance to the functions $Y= \pm\lceil X / p\rceil$ and where the path starts at the vertex with coordinates $(p, 0)$. In this case the paths are counted by a generating function $G_{p}(a, b)=\sum_{i=0}^{p-1} g_{i}(a, b)$ where $g_{i}(a, b)$ generates paths of length $i \bmod p$ and satisfies the set of coupled recurrance equations with a derivative term:

$$
\begin{aligned}
& g_{0}=a b+t(a / b+b / a) g_{p-1} \\
& g_{1}=t\left(a^{2}+b^{2}\right) g_{0}-t a^{2} g_{0}(a, 0)-t b^{2} g_{0}(0, b) \\
& g_{2}=t(a / b+b / a) g_{1}-t a\left[\frac{\partial g_{1}}{\partial b}\right]_{b=0}-t b\left[\frac{\partial g_{1}}{\partial a}\right]_{a=0} \\
& g_{3}=t(a / b+b / a) g_{2}-t a\left[\frac{\partial g_{2}}{\partial b}\right]_{b=0}-t b\left[\frac{\partial g_{2}}{\partial a}\right]_{a=0} \\
& \ldots=\ldots \\
& g_{p-1}=t(a / b+b / a) g_{p-2}-t a\left[\frac{\partial g_{p-2}}{\partial b}\right]_{b=0}-t b\left[\frac{\partial g_{p-2}}{\partial a}\right]_{a=0}
\end{aligned}
$$

Putting $p=2$ in the above recovers the recurrences in equation (5), if the fact that $g_{1}(a, 0)=g_{1}(0, b)=0$ is used.

Putting $a=b=1$, writing $G=\sum_{i=0}^{p-1} g_{i}$ and defining the boundary terms $\Delta g_{0}(a, 0)=0, \Delta g_{1}(a, 0)=g_{1}(a, 0)$ and $\Delta g_{j}(a, 0)=\left[\frac{\partial g_{j}}{\partial b}\right]_{b=0}$ for $j=2,3, \ldots, p-1$ and similarly for $\Delta g_{j}(0, b)$, show that by summing the above recurrances one obtains

$$
G=1+2 t G-\sum_{i=0}^{p-1}\left(\Delta g_{i}(1,0)+\Delta g_{i}(0,1)\right) .
$$

The boundary terms $\sum_{i=0}^{p-1}\left(\Delta g_{i}(1,0)+\Delta g_{i}(0,1)\right)$ represent the generating function of paths starting at the point $(p, 0)$ and ending within one step from the lines $Y= \pm X / p$. Such paths of length $2 p n$ must give almost (within a constant) $(p+1) n$ North East steps. The number of these paths grows at the exponential rate $\lambda=\left((2 p)^{2 p} /(p-1)^{p-1}(p+1)^{p+1}\right)^{n}$. Hence the generating function of these paths is convergent inside the circle of radius $1 / \lambda^{1 / 2 p}$. One can show that this is strictly greater than $1 / 2$, hence all the boundary generating functions $\Delta g_{i}(1,0)$ and $\Delta g_{i}(0,1)$ of paths ending near the boundary of the wedge are convergent on the disk with radius strictly greater than $1 / 2$.

Thus, rearranging equation (46) to get

$$
G=\frac{1-\sum_{i=0}^{p-1}\left(\Delta g_{i}(1,0)+\Delta g_{i}(0,1)\right)}{1-2 t}
$$

the dominant singularity in $G$ is at $t=1 / 2$ and is a simple pole. The subdominant singularities give terms growing to exponential order $\lambda^{n+o(n)}$ where

$$
\lambda=\left(\left(2 p /(p-1)^{(p-1) / 2 p}(p+1)^{(p+1) / 2 p}\right)^{n}\right)
$$

In other words,

$$
c_{n}^{(p)}=A_{p} 2^{n}+O\left(\lambda^{n}\right) .
$$

Putting $p=2$ produces the leading order term and the order of the next term obtained in equation (45). 


\section{Conclusions}

The main results in this paper are given by equations (26) and (45). We have shown that the model of fully directed paths in the wedge $V_{2}$ in figure 3 can be solved using the iterated kernel method. This model is the second in a sequence of models in the wedge $V_{p}$, for $p \in\{1,2,3, \ldots\}$ and recurrences for $p>2$ is given in section 2.4 (when $p=1$ the model is trivial, and $c_{n}=2^{n}$ ). We proved that the number of fully directed path of length $n$ in $V_{2}$ increases exponentially at the rate $C_{0} 2^{n}$, plus subdominant terms which are also exponentials. In addition, we showed that $C_{0}$ (the coefficient of the leading order term) can be determined to high accuracy.

In the more general wedge $V_{p}$, it may be shown, using the techniques in reference [13], that the number of paths of length $n$ is $c_{n}^{(p)}=C_{0}^{(p)} 2^{n}+$ lesser terms. In this event $C_{0}^{(p)}$ is known for $p=1\left(C_{0}^{(1)}=1\right)$ and to high accuracy for $p=2\left(C_{0}^{(2)}=0.678740 \ldots\right)$. No estimates exist for other values of $p$.

The "physical" root $\beta_{0}(a)$ of the quartic kernel (see equation (27)) counts directed paths from $(2,0)$ (see figure 3) above the line $Y=-X / 2$ and with last vertex in the line $Y=-X / 2$. By reversing the horizontal direction, this is also equal to the number of paths from the origin above the line $Y=X / 2$ and with last vertex in the line $Y=X / 2$. The generating function in equation (25) is an alternating series of products of compositions of $\beta_{0}(a)$, suggesting that that $g_{0}(a, 0)$ is "constructed" by an inclusion-exclusion process of directed paths above the lines $Y=-X / 2$ and $Y=X / 2$.

These observations suggest that a combinatorial explanation for the generating function $g_{0}(a, b)$ in equation (26) might be possible in terms of paths "bouncing off" the boundaries of the wedge $V_{2}$. We have not been able to find such an explanation. We leave this question open, but do observe that an explicit explanation in this case might give clues about the nature of the generating function of models in more general wedges, including the models of directed paths in the wedges $V_{p}$ for $p>2$ formed by the lines $Y= \pm X / p$, as well as in the half-wedges formed by the $X$-axis and the lines $Y=X / p$. Such models in half-wedges will be more difficult to solve, since the symmetric nature of $V_{2}$ plays a key role in writing down equation (26) and then determining $C_{0}$ by examining the singularities in equation (40).

Finally, our models in the wedge $V_{p}$ map to the well-known problem of random walks with given step-sets in the quarter plane (the first quadrant). These have been studied for example in references [1, 2, 23]. The models in this paper correspond to models with starting vertex $(p-1, p-1)$ and step set $\{(1-p, p+1),(p+1,1-p)\}$. For $p=2$, this produces the generalised knight's walk model with step set $(-1,3)$ and $(3,-1)$ [21]. The knight's walk problem itself (with step set $\{(-1,2),(2,-1\}$ ) and starting vertex $(1,1)$ was studied in reference [2, where it was shown that its generating function is not holonomic (or $D$-finite).

Acknowledgments: EJJvR and AR are supported by Discovery Grants from NSERC (Canada).

\section{Bibliography}

[1] Bousquet-Mélou M 2001 Counting Walks in the Quarter Plane. In Mathematics and Computer Science: Algorithms, Trees, Combinatorics and probabilities, Trends in Mathematics 49-67 (Birkhauser). 
[2] Bousquet-Mélou M and Petkovšek M 2003 Walks Confined in a Quadrant are not always Dfinite. Theoretical Computer Science 307(2) 257-273.

[3] Brak R, Guttmann A J and Whittington S G 1991 On the Behaviour of Collapsing Linear and Branch Polymers. J. Math. Chem. 8 255-267.

[4] Brak R, Guttmann A J and Whittington S G 1992 A Collapse Transition in a Directed Walk Model. J. Phys. A: Math. Gen. 25 2437-2446.

[5] Delest M-P and Viennot X G 1984 Algebraic Languages and Polyominoe Enumeration. Theor. Comp. Sci. 34 169-206.

[6] Duchon P 2000 On the Enumeration and Generation of Generalized Dyck Words. Disc. Math. 225(1-3) 122-135.

[7] Dvoretzky A and Motzkin T 1947 A Problem in Arrangements. Duke Math. J. 14 305-313.

[8] Feller W 1968 An Introduction to Probability and its Applications. Wiley (New York).

[9] Flory P J 1955 Statistical Thermodynamics of Semi-Flexible Chain Molecules. Proc. Roy. Soc. (London) A 234 60-73.

[10] Flory P J 1969 Statistical Mechanics of Chain Molecules. Wiley Interscience (New York).

[11] Gessel I M 1986 A Probablistic Method for Lattice Path Enumeration. J. Stat. Plan. and Interfer. 14 49-58.

[12] Hammersley J M and Morton K W 1954 Poor Man's Monte Carlo. J. Roy. Stat. Soc. B 16 23-38.

[13] Hammersley J M and Whittington S G 1985 Self-avoiding Walks in Wedges. J. Phys. A: Math. Gen. 18 101-111.

[14] Hille E 1948 Functional Analysis and Semi-Groups. AMS Colloquim Publications 31. American Mathematical Society (Providence, Rhode Island).

[15] Janse van Rensburg E J 1999 Adsorbing Staircase Walks and Polygons, Annals of Combinatorics 3 451-473.

[16] Janse van Rensburg E J 2000 The Statistical Mechanics of Interacting Walks, Polygons, Animals and Vesicles. Oxford Lecture Series in Mathematics and its Applications 18. Oxford University Press (Oxford).

[17] Janse van Rensburg E J 2005 Square Lattice Directed Paths Adsorbing on the Line $Y=q X$, Journal of Statistical Mechanics: Theory and Experiment (September 2005), P09010.

[18] Janse van Rensburg E J 2005 Adsorbing Bargraph Paths in a $q$-Wedge, Journal of Physics A: Mathematical and General 38, 8505-8525. Corrigendum (2006): Journal of Physics A: Mathematical and General 39, 3847.

[19] Janse van Rensburg E J 2006 Forces in Motzkin Paths in a Wedge, Journal of Physics A: Mathematical and General 39, 1581-1608.

[20] Janse van Rensburg E J and Le Y 2005 Forces in Square Lattice Directed Paths in a Wedge, Journal of Physics A: Mathematical and General 38, 8493-8503.

[21] Janse van Rensburg E J, Prellberg T and Rechnitzer A 2007 Partially Directed Paths in a Wedge. To appear in J. Comb. Theory, Ser. A.

[22] Madras N and Slade G 1993 The Self-Avoiding Walk. Birkhäuser (Boston).

[23] Mishna M and Rechnitzer A 2007 Two Non-Holonomic Lattice Walks in the Quarter Plane. Arxiv Preprint math.CO/0701800.

[24] Petkovšek M and Bousquet-Mélou M 2000 Linear Recurrances with Constant Coefficients: the Multivariate Case. Disc. Math. 225(1-3) 51-75.

[25] Prellberg T and Brak R 1995 Critical Exponents from Non-Linear Functional Equations for Partially Directed Cluster Models. J. Stat. Phys. 78 701-30.

[26] Prellberg T and Owczarek A L 1995 Stacking Models of Vesicles and Compact Clusters. J. Stat. Phys. 80 755-79.

[27] Privman V, Forgacs G and Frisch H L 1988 New Solvable Model of Polymer Chains Adsorbing on a Surface. Phys. Rev. B 37 9897-900.

[28] See http://www.research.att.com/ njas/sequences/ 